\title{
Intensive Care Unit and Hospital Outcomes of Patients Admitted with Blastomycosis: A 14-Year Retrospective Study
}

\section{Yahya Almodallal}

Mayo Clinic Rochester: Mayo Clinic Minnesota

Adham K Alkurashi

Mayo Clinic Rochester: Mayo Clinic Minnesota

Hasan Ahmad Hasan Albitar

Mayo Clinic: Mayo Clinic Minnesota

Hussam Jenad

Mayo Clinic Rochester: Mayo Clinic Minnesota

\section{Suartcha Prueksaritanond}

HSHS Saint John's Hospital

\section{Rudy Tedja}

Renown Health

\section{Bibek Pannu}

Cook County Hospital: John H Stroger Hospital of Cook County

\section{Prashant Jagtap}

HSHS Saint John's Hospital

vivek iyer ( $\nabla$ vivekni05@gmail.com )

Mayo Clinic Minnesota https://orcid.org/0000-0001-6441-9319

\section{Research Article}

Keywords: ICU, Blastomycosis, patients, ECMO

Posted Date: February 24th, 2021

DOI: https://doi.org/10.21203/rs.3.rs-220663/v1

License: (c) (i) This work is licensed under a Creative Commons Attribution 4.0 International License. Read Full License 


\section{Abstract}

Introduction: Blastomycosis is an uncommon; potentially life threatening granulomatous fungal infection. The aim of this study is to report hospital and intensive care unit (ICU) outcomes of patients admitted with blastomycosis.

Methods: All patients admitted for treatment of blastomycosis at the Mayo Clinic-Rochester, Minnesota between 01/01/2006 and 09/30/2019 were included. Demographics, comorbidities, clinical presentation, ICU admission, and outcomes were reviewed.

Results: A total of 84 Patients were identified with 93 unique hospitalizations primarily for blastomycosis. The median age at diagnosis was 49 (IQR 28.1-65, range: 6-85) years and 56 (66.7\%) were male. The most frequent comorbidities included hypertension $(n=28,33.3 \%)$; immunosuppressed state $(n=25,29.8 \%)$ and diabetes mellitus $(n=21,25 \%)$. The lungs were the only organ involved in 56 $(66.7 \%)$ cases and the infection was disseminated in 19 (22.6\%) cases.

A total of 29 patients (34.5\%) underwent ICU admission due to complications of blastomycosis. ICU related events included mechanical ventilation $(n=21,25 \%)$, acute respiratory distress syndrome (ARDS) $(n=13,15.5 \%)$, tracheostomy $(n=9,10.7 \%)$, renal replacement therapy $(n=8,9.5 \%)$, and extracorporeal membrane oxygenation (ECMO) $(n=4,4.8 \%)$. A total of 12 patients $(14.3 \%)$ died in the hospital; all of whom had undergone ICU admission. In-hospital mortality was associated with renal replacement therapy (RRT) $(P=0.0255)$.

Conclusions: Blastomycosis is a serious, potentially life-threatening infection that results in significant morbidity and mortality with a $34.5 \%$ ICU admission rate. Renal replacement therapy was associated with in-hospital mortality.

\section{Introduction:}

Blastomycosis is an uncommon granulomatous fungal infection caused by inhalation of spores of dimorphic fungi of the genus Blastomyces. It is most common in North America with incidence rates ranging from 0.3 to 41.9 cases per 100,000 [1-4]. It is considered endemic in the central and southeastern parts of the United States. The lungs are considered the most common site of infection [5]. However, disseminated disease can occur; particularly in immunocompromised patients who tend to develop a more aggressive course of the disease, with earlier dissemination and higher mortality rates [6-8].

Previous studies have shown that $9-23 \%$ of patients require treatment in the ICU with a mortality rate of around $40-44 \%[9,10]$. Although antifungals represent the cornerstone in the management of blastomycosis, there is paucity in data regarding adjunct interventions, including corticosteroids and extracorporeal membrane oxygenation (ECMO), as well as outcomes of patients admitted to the ICU. We 
hereby present our center's experience and discuss the characteristics and outcomes of patients admitted to ICU for management of blastomycosis.

\section{Methods:}

This retrospective study was approved by our institutional review board.

Using the electronic medical record system, we identified all patients who received inpatient management for blastomycosis at the Mayo Clinic in Rochester, Minnesota between 01/01/2006 and 09/30/2019. Only those with hospital admission/s directly related to blastomycosis were included. Data regarding demographics, baseline characteristics including comorbidities and immunosuppressive conditions and medications, diagnostic methods, treatment, ICU admission, and outcomes were analyzed. Acute respiratory distress syndrome (ARDS) was defined and graded according to the Berlin Criteria [11].

\section{Statistical Analysis:}

The data were collected and analyzed using JMP® 14.1.0 (SAS) statistical software. Nominal variables were expressed as frequencies and percentages and compared using Chi square test. Continuous variables were expressed as median (interquartile range, and minimum-maximum range) as they were not normally distributed. The Wilcoxon Rank test was used to compare continuous variables. Factors predicting the outcome were analyzed using the time to event (Kaplan Meier curves) and Nominal logistic regression. $P$-values less than 0.05 were considered statistically significant.

\section{Results:}

\section{Baseline Characteristics (Table 1):}


Table 1

Demographics, and Disease Specifics

\begin{tabular}{|c|c|c|c|c|}
\hline & $\begin{array}{l}\text { Overall } \\
\text { cohort } \\
(n=84)\end{array}$ & $\begin{array}{l}\text { Non-ICU } \\
\text { cohort } \\
(n=55)\end{array}$ & $\begin{array}{l}\text { ARDS ICU cohort } \\
(n=13)\end{array}$ & $\begin{array}{l}\text { Non-ARDS ICU cohort } \\
(n=16)\end{array}$ \\
\hline $\begin{array}{l}\text { Age at time of diagnosis } \\
\text { (years) }\end{array}$ & $49(6-85)$ & $43(6-82)$ & $53(26-73)$ & $63(27-85)$ \\
\hline Male sex & $66.7 \%$ & $65.4 \%$ & $69.2 \%$ & $68.7 \%$ \\
\hline Race & $79.76 \%$ & $78 \%$ & $69 \%$ & $94 \%$ \\
\hline - White & $4.76 \%$ & $4 \%$ & $15 \%$ & $0 \%$ \\
\hline - Black & $15.48 \%$ & $18 \%$ & $16 \%$ & $6 \%$ \\
\hline - Other & $54.76 \%$ & $60 \%$ & $46 \%$ & $44 \%$ \\
\hline Place of residence & $15.5 \%$ & $16 \%$ & $23 \%$ & $6 \%$ \\
\hline - Minnesota & $11.8 \%$ & $4 \%$ & $15 \%$ & $6 \%$ \\
\hline •lowa & $17.94 \%$ & $20 \%$ & $16 \%$ & $44 \%$ \\
\hline \multicolumn{5}{|l|}{ - Wisconsin } \\
\hline \multicolumn{5}{|l|}{ - Other } \\
\hline Comorbidities & $33.3 \%$ & $31 \%$ & $23 \%$ & $50 \%$ \\
\hline - Hypertension & $29.8 \%$ & $27 \%$ & $31 \%$ & $38 \%$ \\
\hline \multicolumn{5}{|l|}{ - Immunosuppression } \\
\hline - Diabetes mellitus & $25 \%$ & $27 \%$ & $19 \%$ & $23 \%$ \\
\hline
\end{tabular}




\begin{tabular}{|c|c|c|c|c|}
\hline & $\begin{array}{l}\text { Overall } \\
\text { cohort } \\
(n=84)\end{array}$ & $\begin{array}{l}\text { Non-ICU } \\
\text { cohort } \\
(n=55)\end{array}$ & $\begin{array}{l}\text { ARDS ICU cohort } \\
(n=13)\end{array}$ & $\begin{array}{l}\text { Non-ARDS ICU cohort } \\
(n=16)\end{array}$ \\
\hline - Malignancy & $23.8 \%$ & $22 \%$ & $31 \%$ & $25 \%$ \\
\hline - ESRD & $11.9 \%$ & $9 \%$ & $8 \%$ & $25 \%$ \\
\hline - COPD & $11.9 \%$ & $11 \%$ & $8 \%$ & $19 \%$ \\
\hline - Organ transplant or & $8.3 \%$ & $9 \%$ & $15.4 \%$ & $0 \%$ \\
\hline Hut & $13.1 \%$ & $12.7 \%$ & $15.4 \%$ & $12.5 \%$ \\
\hline IRD & $1.2 \%$ & $2 \%$ & $0 \%$ & $0 \%$ \\
\hline Sirrberio & $2.38 \%$ & $0 \%$ & $0 \%$ & $13 \%$ \\
\hline 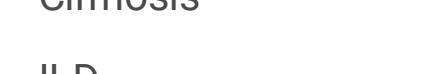 & $3.5 \%$ & $2 \%$ & $0 \%$ & $6 \%$ \\
\hline & $77.4 \%$ & $75 \%$ & $77 \%$ & $87 \%$ \\
\hline - Local & $22.6 \%$ & $25 \%$ & $23 \%$ & $13 \%$ \\
\hline - Disseminated & & & & \\
\hline Systems affected & $88 \%$ & $89 \%$ & $100 \%$ & $75 \%$ \\
\hline - Pulmonary & $15 \%$ & $18 \%$ & $8 \%$ & $13 \%$ \\
\hline - Skin & $8 \%$ & $9 \%$ & $0 \%$ & $13 \%$ \\
\hline - CNS & $7 \%$ & $9 \%$ & $0 \%$ & $6 \%$ \\
\hline - Bones & $2 \%$ & $2 \%$ & $8 \%$ & $0 \%$ \\
\hline - Kidneys & & & & \\
\hline
\end{tabular}

A total of 84 patients were identified with 93 unique hospitalizations primarily for blastomycosis. Of all 84 patients, $66.7 \%(n=56)$ were male and $80.0 \%(n=67)$ were white. The median age at diagnosis was 49.0 (IQR 28.1-65.0, range: 6-85) years.

Comorbidities at first admission included hypertension ( $n=28,33.3 \%)$; immunosuppressed state $(n=25$, $29.8 \%)$, diabetes mellitus $(n=21,25 \%)$, malignancy $(n=20,23.8 \%$ including 8 patients with active malignancy at time of diagnosis and 12 with a prior history of malignancy), end stage renal disease (ESRD, $n=10,11.9 \%$ ), chronic obstructive pulmonary disease (COPD, $n=10,11.9 \%$ ), history of solid organ and/or hematopoietic stem cell transplant (HSCT, $n=7,8.3 \%)$, autoimmune condition $(n=11,13.1 \%$; rheumatoid arthritis in 3 patients, hypothyroidism in 4 patients and vitiligo in 2 patients, and myositis in 2 patients), interstitial lung disease (ILD, $n=3,3.5 \%)$, liver cirrhosis $(n=2,2.4 \%)$, and inflammatory bowel disease (IBD, $n=1,1.2 \%$ ). 
Of the 84 patients, blastomycosis affected only one organ in 65 cases $(77.4 \%)$, and was disseminated in $19(22.6 \%)$. The infection most commonly involved the respiratory system in 74 cases (88\%), followed by skin involvement in 13 cases (15\%) and the central nervous system (CNS) in 7 cases (8\%). Other involved organs included bones $(n=6(7 \%))$, kidneys $(n=2(2 \%))$, heart, and spleen.

In those with pulmonary involvement, the disease most commonly affected the right lower lobe $(n=18$, $24.3 \%)$ followed by the right upper lobe $(n=17,23.0 \%)$, and was bilateral in 15 patients $(20.3 \%)$.

\section{Diagnosis And Treatment (table 2):}




\section{Diagnostic method :}

Histopathology

- Positive

- Negative

- Not performed

Culture

- Positive

- Negative

- Not performed

Serology*

- Positive

- Negative

- Not performed

Urinary antigen

- Positive

- Negative

- Not performed

Bronchoalveolar lavage

Performed

- TNC (range)

- AM

- Lymphocyte percentage

- Neutrophil percentage

- Eosinophil percentage
$70.3 \%$

$23.1 \%$

$6.6 \%$

$75 \%$

$14 \%$

$11 \%$

$48.4 \%$

$36.6 \%$

$15 \%$

$34.8 \%$

$27.2 \%$

$38 \%$
Median (range)

$51.2 \%$

56.4 (23.8-605.1)

53.5 (17.5-84.75) \%

$2(1-12) \%$

$40(9-88.5) \%$

$2(1-2) \%$

* refers to antibody immunodiffusion assay, antibody enzyme-linked immunoassay, or both. TNC: total nucleated cell count, AM: Alveolar Macrophages.

The diagnosis of blastomycosis was established with culture in and/or histopathologic examination in $76(90.5 \%)$. In the remaining 8 (9.5\%), the diagnosis was established by serologic testing.

Bronchoalveolar lavage (BAL) was performed in 43 patients (51.2\%); culture was obtained in 39 of those 
(90.7\%) and was positive in 35 patients (90.0\%). All cases were reviewed and confirmed by multidisciplinary teams including Infectious Diseases specialists.

\section{Icu Admission, Management And Outcomes:}

The median hospital stay was 8 (IQR 4-17) days for the study cohort. A total of 29 (34.5\%) admissions were managed in the ICU with a median ICU stay of 6 (IQR 1-21) days.

A seasonal variation in the rate of admission to the ICU were noted; patients who presented in winter were six-times more likely to be admitted to the ICU when compared to those presented in spring $P=0.028$.

Among those admitted to the ICU $(n=29) ; 13(44.8 \%)$ had ARDS. A total of $14(48.3 \%)$ patients required non-invasive mechanical ventilation and $20(69 \%)$ required invasive mechanical ventilation (7 (35\%) initiated on admission and 13 (65\%) failed non-invasive mechanical ventilation). Tracheostomy was performed in 9 patients (33.3\%) after a median of 7.5 days (4-28) days of invasive mechanical ventilation. Further details regarding respiratory support are summarized in Table 3. 
Table 3

ICU interventions and respiratory support

\begin{tabular}{|llll|}
\hline & $\begin{array}{l}\text { Overall } \\
(\mathbf{N}=29)\end{array}$ & $\begin{array}{l}\text { ARDS ICU cohort } \\
(\mathbf{n}=\mathbf{1 3})\end{array}$ & $\begin{array}{l}\text { Non-ARDS ICU cohort } \\
(\mathbf{n}=\mathbf{1 6})\end{array}$ \\
\hline Median ICU stay period & 6 days & 8 days & 2.5 days \\
- Vasopressors & $75.9 \%$ & $100 \%$ & $56.2 \%$ \\
- Number of vasopressors & $2(1-4)$ & $3(1-4)$ & $2(1-4)$ \\
- Patients required one & $7(31.8 \%)$ & $3(23 \%)$ & $4(44.4 \%)$ \\
vasopressors & $15(68.2 \%)$ & $10(77 \%)$ & $5(55.6 \%)$ \\
- Patients required two or more & $62 \%$ & $92.3 \%$ & $62.5 \%$ \\
vasopressors & $44.8 \% \%$ & $100 \%$ & $0 \%$ \\
- Glucocorticoids & $41.4 \% \%$ & $53.8 \%$ & $31.2 \%$ \\
- ARDS & $33.3 \% \%$ & $46.1 \%$ & $21.4 \%$ \\
- Renal replacement therapy & $14.8 \%$ & $33.3 \%$ & $0 \%$ \\
- Tracheostomy & $21(11-183)$ & $21(11-183)$ & - \\
- ECMO & days & $46.1 \%$ & $37.5 \%$ \\
o Median duration of use (range) & $41.4 \%$ & & \\
Mortality Rate & & &
\end{tabular}

* refers to continuous positive airway pressure (CPAP), bilevel positive airway pressure (BIPAP), or both. \# Data was found for 9 patients only. 


\begin{tabular}{|c|c|c|c|}
\hline & $\begin{array}{l}\text { Overall } \\
(\mathrm{N}=29)\end{array}$ & $\begin{array}{l}\text { ARDS ICU cohort } \\
(n=13)\end{array}$ & $\begin{array}{l}\text { Non-ARDS ICU cohort } \\
(n=16)\end{array}$ \\
\hline Respiratory support in ICU: & $79.3 \%$ & $92.3 \%$ & $68.7 \%$ \\
\hline - Nasal cannula or face mask & $48.3 \%$ & $76.9 \%$ & $25 \%$ \\
\hline \multirow{4}{*}{$\begin{array}{l}\text { - Non-invasive mechanical } \\
\text { ventilation* } \\
\text { - Invasive mechanical ventilation } \\
\text { o Median duration of use (range) }\end{array}$} & $69 \%$ & $100 \%$ & $43.7 \%$ \\
\hline & \multirow{3}{*}{$\begin{array}{l}\text { days } \\
93.3(34- \\
325)\end{array}$} & $8(1.5-57)$ & $7(4-41)$ \\
\hline & & $83.7(34-325)$ & $130.8(74-230)$ \\
\hline & & $81(30-176)$ & $104.2(74-194)$ \\
\hline •PF ratio: median (range) & \multirow{3}{*}{$\begin{array}{l}83.7(30- \\
194) \\
0.875(0.24- \\
1)\end{array}$} & $1(0.24-1)$ & $0.6(0.4-1)$ \\
\hline ventilation & & $1(0.5-1)$ & $0.6(0.4-1)$ \\
\hline o Lowest during ventilation & & $14(10-20)$ & $6(5-12)$ \\
\hline • FiO2: median (range) & $0.95(0.4-1)$ & & \\
\hline $\begin{array}{l}\text { o Highest within first } 24 \text { hours of } \\
\text { ventilation }\end{array}$ & $10(5-20)$ & & \\
\hline \multicolumn{4}{|l|}{ o Highest during ventilation } \\
\hline \multicolumn{4}{|l|}{ • PEEP: median (range) } \\
\hline o Highest during ventilation & & & \\
\hline
\end{tabular}

Vasopressor support was required for $22(75.9 \%)$ patients, of which, 15 (68.2\%) required multiple vasopressors. Four (14.8\%) patients required initiation of ECMO for refractory hypoxemic respiratory failure. Of those, $3(75 \%)$ required veno-venous ECMO and 1 (25\%) required veno-arterial ECMO for concurrent heart failure. The median duration ECMO was required was $21(11-183)$ days. Only 1 patient was successfully weaned from ECMO support while the remaining 3 patients died because of (fulminant pulmonary blastomycosis, disseminated blastomycosis complications, and acute hypoxemic respiratory failure).

Renal replacement therapy (RRT) was required for 12 (41.4\%) patients; of whom, 4 patients were receiving RRT prior to the admission due to ESRD while 8 patients required new initiation of RRT for acute kidney injury. Of the 8 patients who required initiation of RRT, 3 developed chronic kidney disease after discharge and remained on dialysis until the last follow-up.

In addition to antifungal therapy, glucocorticoids were administered in $18(62 \%)$ cases with a median dose of 46 (8-1250) mg prednisone-equivalent per day, for a median duration of use of 6 (1-106) days. 


\section{Mortality In Blastomycosis Patients:}

A total of $25(29.8 \%)$ patients died at the end of follow-up with a total of 12 hospital deaths $(14.3 \%)$ directly related to blastomycosis. The overall in-hospital, ICU and ARDS mortality rates were $14.3 \%, 41.4 \%$ and $46.1 \%$, respectively.

Using univariate analysis, a positive correlation was observed between in-hospital mortality and age $>65$ years at the time of diagnosis $(P=0.04)$, and renal replacement therapy $(P=0.03)$, and invasive mechanical ventilation use $(P=0.048)$. However; no correlation was found between steroid use, chronic lung disease, chronic heart disease, number of lobes involved, and ARDS. Multivariable logistic regression could not be done due to the small sample size. For 1-month mortality, a positive correlation was observed between mortality and ICU admission $(P=0.0007)$, age more than 65 at time of diagnosis $(P=$ 0.01), Renal replacement therapy $(P=0.03)$, and invasive mechanical ventilation use $(P=0.048)$. Multivariable logistic regression could not be done due to small sample size.

\section{Discussion:}

Previous studies on blastomycosis have largely focused on the epidemiological aspect of the disease while a few studied the clinical characteristics and outcomes. This study investigates the hospital and ICU outcomes of 84 patients with confirmed diagnosis of blastomycosis admitted to a tertiary care center.

Our study found a significantly lower rate of isolated pulmonary involvement $(67 \%)$ as compared to previous studies from Ontario, Canada and Indiana, USA (90\%). [12] [10] The fact that more patients in our study presented with disseminated disease could be a potential explanation for the higher ICU admission rate in our study (34.5\%) as compared to previous cohorts $(10 \%$ and $23 \%)$ [10, 12$]$. Similarly, the higher mortality rate (14.3\%) in our cohort may also have been related to the higher rates of disseminated disease at time of presentation.

Schwartz et al. [9] focused mainly on outcomes of patients with ARDS secondary to blastomycosis. They studied the clinical course of 43 patients with ARDS in Manitoba, Canada. In their study, $98 \%$ of patients were intubated (compared to $100 \%$ intubation rate in our study), $11 \%$ received ECMO ( $14.8 \%$ in our study), while $51 \%$ received steroids ( $62 \%$ in our study). The mortality rate reported in their study $(40 \%)$ was similar to that reported in this study (46.1\%). Our data also confirmed a significantly higher mortality rate in patients with blastomycosis related ARDS (46.1\%) as compared to those without ARDS (37.5\%). Despite the higher mortality rate in blastomycosis cases with ARDS, it is worth mentioning that it remains similar to mortality rate seen in ARDS secondary to other causes in general [16]. The role of adjunct therapies including corticosteroids and ECMO for the management of blastomycosis remains to be determined. Schwartz et al. [9], reported a 100\% survival for the 4 ARDS cases treated with ECMO, whereas in our cohort we noted a $75 \%$ mortality rate for ECMO treated blastomycosis related ARDS patients. 
Our study has a number of limitations including its retrospective design, single center experience and small sample size, all of which could limit its external validity. However, given the infrequency of this condition, a retrospective study design appears to be the only feasible option to study this condition. Despite the small sample size, ours is one of the few studies that have focused exclusively on hospital and ICU outcomes of blastomycosis patients.

\section{Conclusion:}

Blastomycosis is a serious, potentially life-threatening infection that results in significant morbidity and mortality and high ICU admission rates. Invasive mechanical ventilation use, age more than 65 at time of diagnosis, and renal replacement therapy were all associated with in-hospital mortality. Clinicians in areas endemic with blastomycosis should be aware of this infection, and the crucial role of early recognition and treatment in its management. Future collaboration between centers specialized in management of blastomycosis is crucial to study this disease on a larger scale and help draw more conclusive associations and recommendations.

\section{Abbreviations}

ARDS: acute respiratory distress syndrome

BAL: Bronchoalveolar lavage

COPD: chronic obstructive pulmonary disease

CNS: central nervous system

ECMO: extracorporeal membrane oxygenation

ESRD: end stage renal disease

HSCT: hematopoietic stem cell transplant

IBD: inflammatory bowel disease

ICU: intensive care unit

ILD: interstitial lung disease

RRT: renal replacement therapy

\section{Declarations}

Author Contribution: Conception and design: Y.A, A.A, H.A, S.P, R.T, B.P, P.J, V.I; Analysis and interpretation: Y.A, A.A, H.A, V.I; Drafting the manuscript for important intellectual content: Y.A, A.A, H.A, H.J, V.I 
Corresponding author: Vivek N. Iyer, M.D, MPH, iyer.vivek@mayo.edu , 200 1st St. SW, Rochester, MN, 55905

Conflict of interest: The authors declare that there is no conflict of interest regarding the publication of this paper.

\section{Acknowledgment: None.}

Summary conflict of interest statements: The authors have no conflicts of interest to report.

Funding information: The authors have no funding sources to report.

\section{References}

1. Brown EM et al., Epidemiology and Geographic Distribution of Blastomycosis, Histoplasmosis, and Coccidioidomycosis, Ontario, Canada, 1990-2015. Emerging infectious diseases, 2018. 24(7): p. $1257-1266$

2. Benedict K et al (2012) Epidemiologic and Ecologic Features of Blastomycosis: A Review. Current Fungal Infection Reports 6(4):327-335

3. Reed KD et al., Ecologic Niche Modeling of Blastomyces dermatitidis in Wisconsin. PLOS ONE, 2008. 3(4): p. e2034

4. Morris SK et al., Blastomycosis in Ontario, 1994-2003. Emerging infectious diseases, 2006. 12(2): p. $274-279$

5. Smith JA, Kauffman CA, Blastomycosis. Proceedings of the American Thoracic Society (2010) 7(3): p. $173-180$

6. Pappas PG et al (1993) Blastomycosis in immunocompromised patients. Medicine 72(5):311-325

7. Recht LD et al (1982) Blastomycosis in immunosuppressed patients. Am Rev Respir Dis 125(3):359362

8. Serody JS et al (1993) Blastomycosis in transplant recipients: report of a case and review. Clin Infect Dis 16(1):54-58

9. Schwartz IS et al (2016) Management and Outcomes of Acute Respiratory Distress Syndrome Caused by Blastomycosis: A Retrospective Case Series. Medicine 95(18):e3538-e3538

10. Azar MM et al (2015) Blastomycosis in Indiana: Clinical and Epidemiologic Patterns of Disease Gleaned from a Multicenter Retrospective Study. Chest 148(5):1276-1284

11. Force* TADT (2012) Acute Respiratory Distress Syndrome: The Berlin Definition. JAMA 307(23):2526-2533

12. Kralt D et al (2009) Clinical characteristics and outcomes in patients with pulmonary blastomycosis. Mycopathologia 167(3):115-124 
13. Chapman SW et al (1997) Endemic blastomycosis in Mississippi: epidemiological and clinical studies. Semin Respir Infect 12(3):219-228

14. Pfister JR et al (2011) Non-rural point source blastomycosis outbreak near a yard waste collection site. Clin Med Res 9(2):57-65

15. Roy M et al (2013) A large community outbreak of blastomycosis in Wisconsin with geographic and ethnic clustering. Clinical infectious diseases 57(5):655-662

16. Bellani G et al (2016) Epidemiology, Patterns of Care, and Mortality for Patients With Acute Respiratory Distress Syndrome in Intensive Care Units in 50 Countries. Jama 315(8):788-800

17. Zambon M, Vincent J-L (2008) Mortality Rates for Patients With Acute Lung Injury/ARDS Have Decreased Over Time. Chest 133(5):1120-1127 